\title{
Establishing the required components for training in ultrasound- guided peripheral intravenous cannulation: a systematic review of available evidence
}

\author{
Fredericus HJ van Loon', Harm J Scholten², Irene van Erp', Arthur RA Bouwman ${ }^{3}$, \\ Angelique TM Dierick-van Daele ${ }^{4}$
}

${ }^{1}$ Department of Technical and Anesthesia Nursing Sciences; Fontys University of Applied Sciences; Department of Anesthesiology, Pain Medicine and Intensive Care; ${ }^{2}$ Department of Anesthesiology, Pain Medicine and Intensive Care, ${ }^{3}$ Department of Signal Processing Systems and Electrical Engineering, TU/e University of Technology; Department of Anesthesiology, Pain Medicine and Intensive Care, ${ }^{4}$ Department of People and Health Sciences; Fontys University of Applied Sciences; Department of Research and Education; Catharina Hospital, Eindhoven, The Netherlands

\begin{abstract}
Ultrasound increases a first-attempt success rate for vascular access when considered by knowledgeable and experienced practitioners. Education and training of these practitioners in ultrasound-guided peripheral intravenous cannulation is becoming increasingly common, although no consensus has been reached regarding its curriculum. The current systematic literature review aims to explore different training modules and components in use, and its efficacy and efficiency in ultrasound-guided peripheral intravenous cannulation in hospitalized adults by different healthcare providers. Database search was performed from January 2009 to December 2018 for publications describing the training or education of healthcare professionals in ultrasound-guided peripheral intravenous cannulation in adult patients. Data-analyses was performed on 23 studies, concluding that competency on ultrasound-guided peripheral intravenous cannulation can be achieved after following a brief training in a fixed curriculum, consisting of a didactic training session, a simulated hands-on component, and is completed after a supervised live-case training. Lectures should focus on ultrasound physics, including the vascular anatomy. The hands-on training included identification of veins on a life model without cannulating, followed by cannulation of veins using a nonhuman tissue model. At the end, supervised cannulation of veins on the upper extremity with an ultrasound-guided technique was performed on live patients to show competency.
\end{abstract}

Keywords: catheterization; peripheral; vascular access devices; ultrasonography; education

\section{Introduction}

Establishing peripheral intravenous access is a critical skill for patient care that can be technically challenging at times. Up to four out of five patients requires a periph-

Received 01.08.2019 Accepted 06.10.2019

Med Ultrason

2019, Vol. 21, No 4, 464-473

Corresponding author: F.H.J. van Loon, MSc.

Catharina Hospital, Department of Anesthesiology, Pain Medicine and Intensive Care Michelangelolaan 2,

5623 EJ Eindhoven, The Netherlands

E-mail: rick.v.loon@catharinaziekenhuis.nl eral intravenous catheter during their stay in the hospital, making peripheral intravenous cannulation the most frequently performed invasive medical procedure [1,2]. Nonetheless, despite its routine nature, peripheral intravenous cannulation is not always successful on the first attempt [3-6]. Using the traditional landmark approach, a success rate of $70 \%$ on the first attempt of peripheral intravenous cannulation was reported in a recently performed meta-analysis, whereas a success rate of $81 \%$ was seen in patients in which the ultrasound-guided technique was used as result of that study.

Over the past two decades, ultrasound has become widely accepted to guide safe and accurate insertion of 
vascular devices in hospitalized patients. Improvements in technology, including miniaturization, have led to the development of pocketsize imaging devices. Ultrasound equipment has become more compact, with good image quality and less expensive, which has facilitated the growth of point-of-care ultrasonography [8]. Although there are many published studies demonstrating the benefits of ultrasound guidance for intravenous cannulation, simply placing an ultrasound probe on a patient's extremity does not ensure success [7]. The physical limitations of the behavior of ultrasound in tissue must be fully understood by the operator, otherwise failure of vascular cannulation will result [9].

To lower the threshold for applying ultrasound guidance during peripheral intravenous cannulation, different healthcare providers need to be trained and gain experience in using this technique $[6,10,11]$. Training and practice will subsequently improve ultrasound use and increase success, at which success using ultrasound for intravenous cannulation is based on appropriate equipment and preparation, optimal vein selection, and effectively using ultrasound to understand and guide needle tip position [8]. Ultrasound physics and transducer properties should be part of the training for understanding ultrasound-related artefacts and pitfalls, as concluded in a previous study by Van Loon et al [6]. Education surrounding the insertion of peripheral intravenous catheters remains undefined because a standardized program for trainees, nor a guideline for supervisors, is lacking in current practice [11]. Understanding and establishing the level of training required for safe insertion procedures and management of ultrasound-guided peripheral intravenous cannulation is therefore the focus of this study [11].

Education and training of practitioners, both physicians and nurses, in ultrasound-guided peripheral intravenous cannulation is becoming increasingly common and key management of the difficult-access patient; however, no consensus has been reached regarding its value $[8,11,12]$. Challenges include gaining better understanding of when and how point-of-care ultrasound can be used effectively, and structuring policy and reimbursement to encourage appropriate and effective use [7]. Therefore, the current systematic literature review aims to explore different training modules and components in use, and its efficacy and efficiency on ultrasound-guided peripheral intravenous cannulation in hospitalized adults by different healthcare providers.

\section{Materials and methods}

This systematic literature review was conducted following the established guidelines from the Preferred Re- porting Items for Systematic Reviews and Meta-Analyses (PRISMA) [6,13].

\section{Search strategy}

Observational and interventional studies describing training or education of healthcare professionals in ultrasound-guided peripheral intravenous cannulation in adults were systematically searched in databases of peer reviewed literature, including PubMed, Clinical Key, Cochrane Library of Clinical Trials, and Trip Database. Manuscripts in the English and Dutch languages as published from January 1, 2009 until December 31, 2018 were included. The early used portable ultrasound machines were hampered by poor image quality, but imaging quality and employability increased from 2010 [8]. For this reason, only studies published in the last ten years were included. Google Scholar was searched for additional literature sources. The primary search criteria included "peripheral intravenous cannulation, peripheral intravenous access, peripheral intravenous catheterization", and were connected by the Boolean "AND" with the terms "ultrasound, ultrasound guidance, ultrasonography, ultrasound-guided", and connected by "AND" to "learning curve, education, training". Medical Subject Headings (MeSH) terms was used if appropriate.

\section{Study selection}

Studies describing the content or components of the training program, as well as studies describing the learning curve of healthcare professionals for ultrasound-guided peripheral intravenous cannulation in adult humans, were included. Studies were excluded for the following reasons: (1) intravenous cannulation on other sites of the body (e.g. central venous) rather than the upper extremity; (2) intravenous insertion of other devices (e.g. central venous catheters, peripheral inserted central venous catheters, dialysis catheters, arterial catheters) rather than short peripheral intravenous catheters; (3) describing the deployment of the ultrasound-guided technique without focusing on the training or learning curve; and (4) if a non-ultrasound-guided technique was used (e.g. traditional approach based on landmarks) [6]. Studies were selected regardless the setting in which the study was performed (e.g. emergency department, surgical operating theatre complex), the applied ultrasound technique (e.g. single- or two-operator, short- or long-axis viewing, dynamic or static technique), or the practitioner included in the study (e.g. nurse or physician).

\section{Outcome measures}

This study focuses on the following outcome measures: elements or content of the training or educational program, as well as the length or duration, covered topics, used materials, preconditions, homework or foreknowledge, the learning curve or punctures needed until 
a stable success rate was reached, final objectives, and certification. This study does not concentrate on the final implementation of an ultrasound-training program in daily practice or educational curricula.

\section{Data extraction}

Two reviewers (F.L. and I.E.) screened eligible studies independently, according to a previous presented strategy on title and abstract, and classified them as being relevant, potentially relevant, or not relevant [6]. Secondly, full-text of the articles that were classified as being relevant were analyzed by both reviewers independently [6]. Hereafter, both reviewers decided individually if the study was eligible or not, based on the inclusion and exclusion criteria [6]. Any discrepancy between the reviewers was resolved with a final decision from a third independent investigator (A.B.) [6]. Eligibility of studies classified as being potentially relevant in the first phase was also decided by an independent investigator (A.B.), after which those studies with a positive final decision were included [6].

\section{Statistical analyses}

Ad-hoc tables will be designed to summarize data from the included studies and show their key characteristics and important questions related to the objectives of this review. After data extraction, reviewers will determine whether a meta-analysis is possible.

\section{Results}

In total, 23 studies were selected and included for final analysis after database review, removal of duplicates, title and abstract screening, and full-text review (fig 1), of which the characteristics are represented in Table I.

The presentation of the results is subdivided into several main topics that are considered relevant for answering the literature search question or supporting the conclusion. In general, training for ultrasound-guided peripheral intravenous cannulation can be divided into a didactic training module, a hands-on training session, and training of skills in life-cases. An overview is given in figure 2 .

\section{Didactic training}

In the didactic training session, lectures are used to support the transfer of knowledge [14]. The didactic training session can be divided mainly into three general themes: ultrasound physics; the ultrasound-guided technique of cannulation; and the vascular anatomy. Within ultrasound physics, subjects such as: basic ultrasound use; the ultrasound probe and probe selection; and the ultrasound machine and knobology of the device, are explained [10,14-24]. Trainees in ultrasound techniques focused on: differentiating veins from arteries, nerves

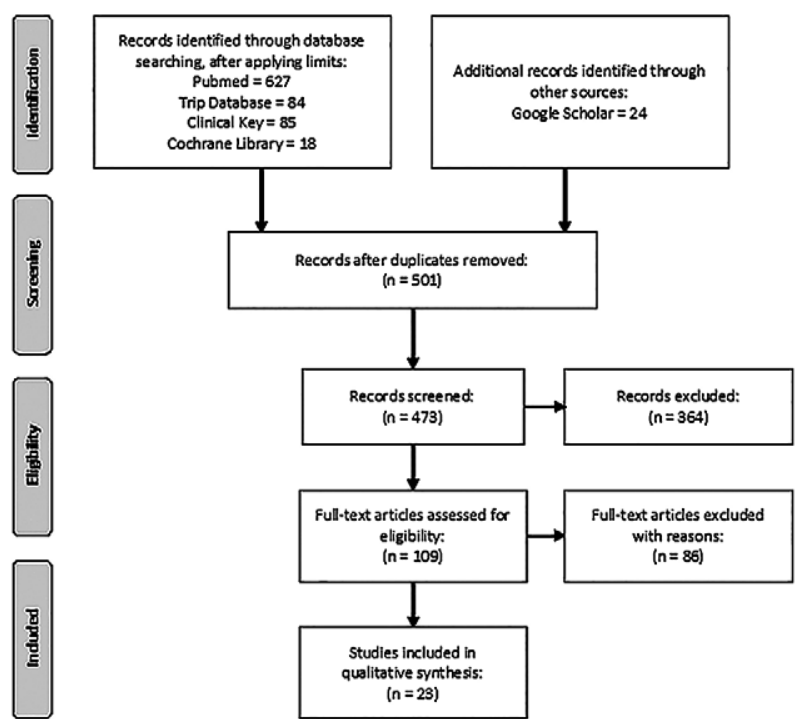

Fig 1. PRISMA flowchart of study selection

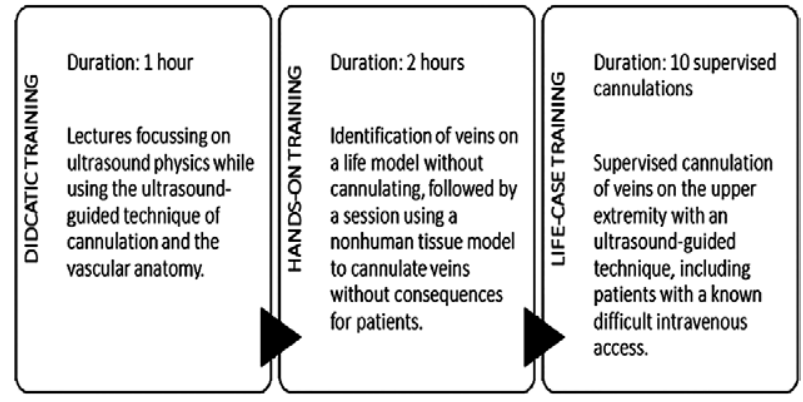

Fig 2. Overview of training sessions, including the components and content, and suggested duration of the sessions.

and muscles; applying venous compression or (color) Doppler to identify venous structures; appropriate selection of the cannulation site, vein and needle entry point; the approach of visualizing the needle or the guidance technique (dynamic short-axis out-of-plane or dynamic long-axis in-plane); and optimization of images, acquisition and screen settings (gain and depth) $[10,14,15,17$ 26]. Theoretical considerations of the anatomy include the vascular anatomy of the upper extremity and physiology of the circulation $[10,14,15,18,20,21,27]$. Besides these three main topics, didactic training covers subjects as disinfection and catheter care, prevention of infections, disinfection and cleaning of the machine, and care of equipment $[14,18,20,24]$. Also, the procedure of peripheral intravenous cannulation, documentation, indications for ultrasound use, and identification and management of complications are included in training programs $[10,14,15,17,19,22,28]$. Some studies included a video demonstration of peripheral intravenous cannulation under ultrasound guidance as part of the training program 
Med Ultrason 2019; 21(4): 464-473 467

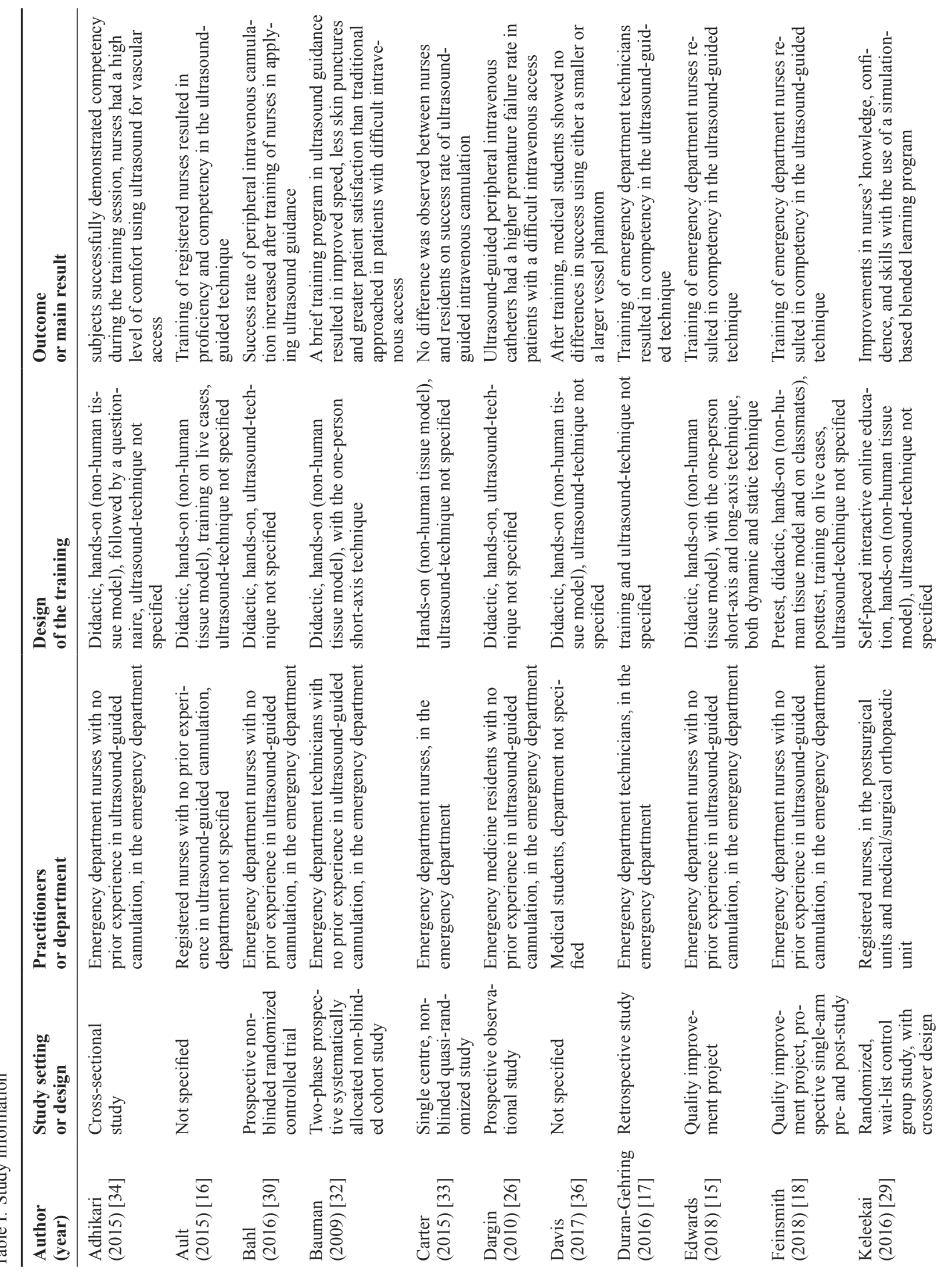




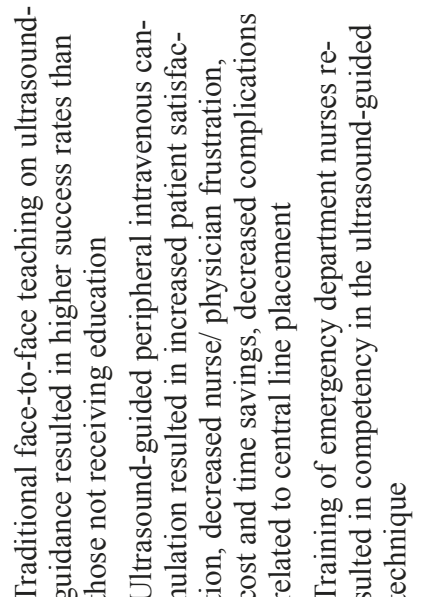

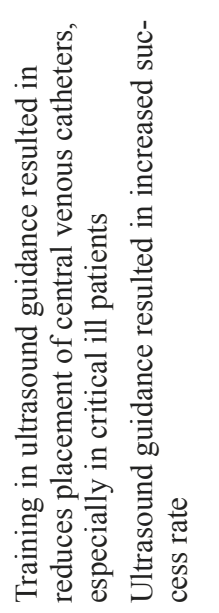

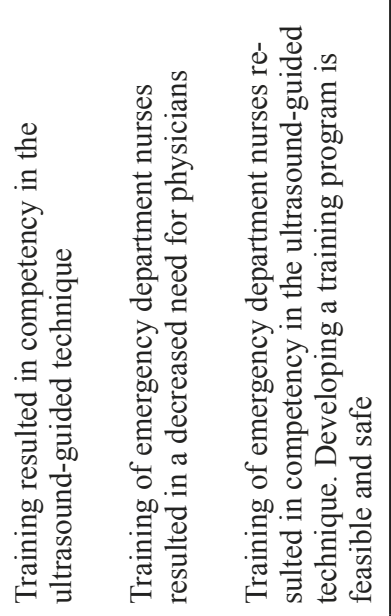

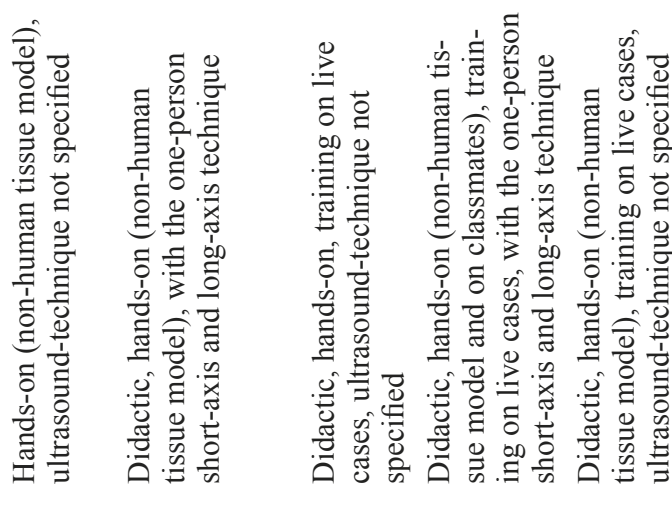

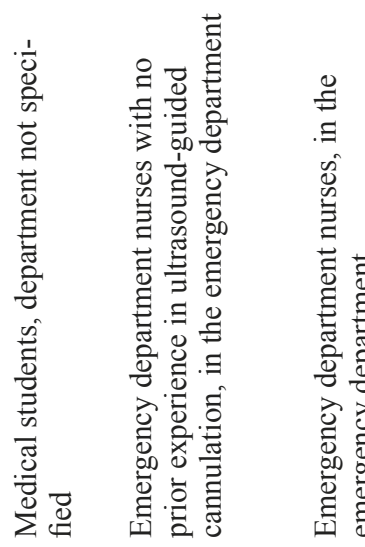

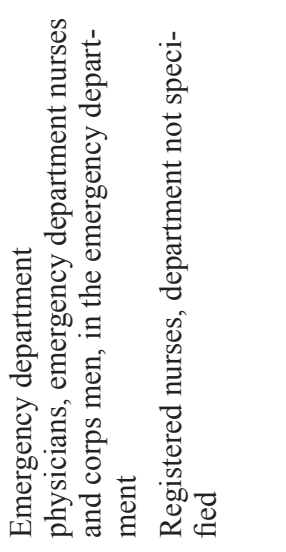

害

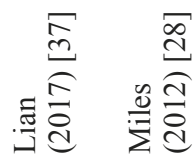

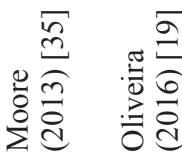

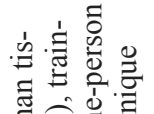

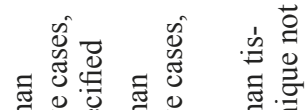

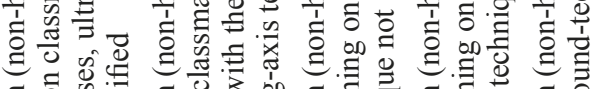

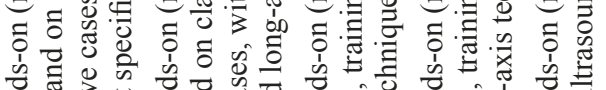

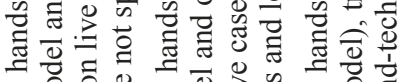

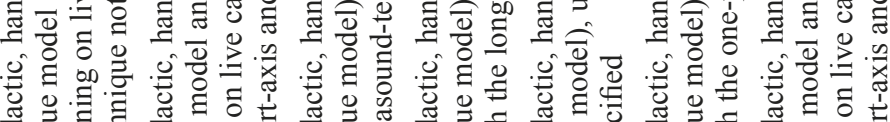

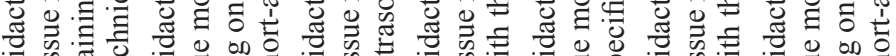

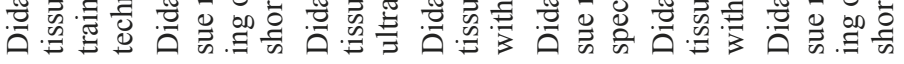

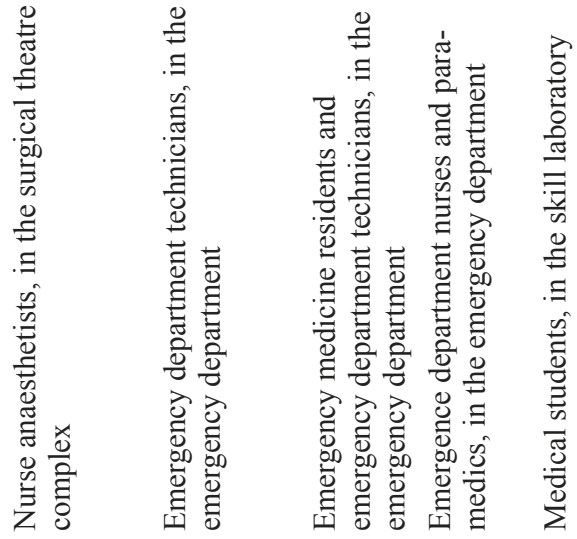

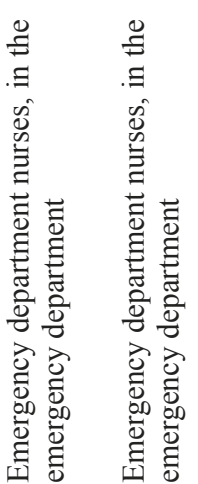

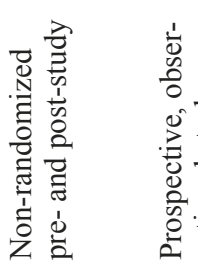

崩

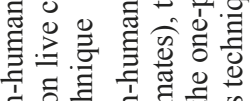

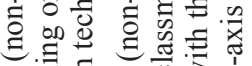

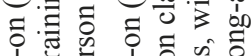

iै $\exists$ के

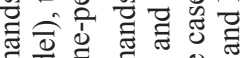

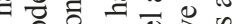

\section{政言}

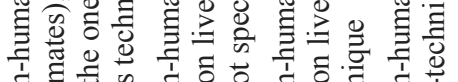


$[14,16,18,29,30]$. The training session as described in the study by Keleekai et al consisted of an online course, including several aforementioned aspects [29].

\section{Hands-on training}

A hands-on training session logically follows the didactic training, requiring trainees to gain and show competency before acting on life cases [15]. A hands-on training session in a simulation setting creates a situation in which trainees can familiarize themselves with the ultrasound machine and equipment [10,14,24,29]. Furthermore, it enables trainees to focus on basic ultrasound acquisition and creating still images, as well as becoming aware of the upper extremity anatomy $[10,14,20,22,24,29,31,32]$. Identifying the anatomy of the upper extremity by tracing veins on a life model without cannulating it allows trainees to appreciate the vein characteristics, and gain eye-hand coordination with probe manipulation [10,14,18-22]. A 1:1 hands-on session using a nonhuman tissue model, to continue, creates a possibility for trainees in emphasizing confirmation and visualizing of the needle tip while deforming the target vein prior to cannulation, the dexterity to insert an intravenous catheter while holding the probe and watching the screen (eye-hand coordination), and visualization of venous cannulation on the ultrasound screen in real-time [10,14-19,21-26,28,31,32]. A simulated training session could also focus on sterility and aseptic techniques without consequences for the patient $[24,27]$. According to Adhikari et al practitioners had a high level of comfort using ultrasound for intravenous cannulation after a focused simulation training session, resulting in the accurate identification of the vascular anatomy and performing ultrasound-guided vascular access [34].

\section{Life-case training}

During life-case training, trainees gain experience and routine in cannulating veins on the upper extremity with an ultrasound-guided technique in human subjects, including patients with a known difficult intravenous access $[14,15,23,29,31]$. Focus of the trainee should be on keeping the needle tip in the ultrasound field while navigating to the vein, perfecting probe control, treading the needle under ultrasound guidance, and attempting cannulation of smaller and deeper veins [24]. According to the statement of Edwards et al the biggest question on determining competency was how many supervised successful procedures needed to be performed before a trainee could function independently [15]. Of the included studies, most recommend 10 supervised attempts in life cases $[15,17,24,27,29]$. Oliveira et al and PartoviDeilami et al found 3 supervised cannulations to be sufficient to show competency, while Duran-Gehring et al and Edwards et al suggest trainees to perform 5 supervised attempts in their training program $[10,15,17,19]$. Additionally, trainees should perform 5 attempts under indirect supervision, with oversight immediately available, according to Edwards et al [15]. However, the study by Moore et at required 25 supervised attempts to produce a success rate of $80 \%$. Stolz et al described in their study that supervision and training must be continued until a trainee reached an individual success rate of $70 \%$ on the first attempt, and for this, 4 supervised attempts were needed [23].

\section{Additional provisions}

Keeping a $\log$ or data collection form can provide support in demonstrating competency, in which the formulated final objective can be leading [16-18,34]. Competency can be determined with testing of the practitioners before and after the training $[16,17,20]$. Duration of training varied between the included studies, from two hours until multiple days $[10,17,22,23,34]$. Didactic session duration ranged between 30 minutes up to 3 hours $[10,14,15,18,20,24-27,29,34]$. The same applied to hands-on training, with the duration of the training varying between 1 and 2 hours [10,15,18,24,25,27,31]. Edwards et al, in the meantime, placed no restrictions on the time needed for the hands-on training, while Keleekai et al described a hands-on training with a duration of 8 hours $[15,29]$.

\section{Effects of training}

As concluded by Lian et al, trainees receiving education on ultrasound-guided venous cannulation performed significantly better than those not receiving education $[35,37]$. Trainees with no prior ultrasound experience can achieve competency on ultrasound-guided peripheral intravenous cannulation when given a dedicated training and closed supervision in a fixed curriculum $[10,14-$ 20,22-24,26,28,29,31]. After completing a brief training program, participants were more successful in obtaining intravenous access using ultrasound guidance in patients with a known difficult venous access when compared to a situation in which the traditional landmark technique of peripheral intravenous cannulation was used $[19,23,29,31]$.

\section{Assumptions for implementation}

Several administrative processes need to be defined and implemented before the implementation of a training program, including the development of a practice statement, procedure guidelines, operational plan, and competency validation [14]. To add to this, an environment with widespread opportunities for ultrasound-guided peripheral intravenous cannulation is recommended for the rapid acquisition of skills [17]. As the number of ultrasound machines in clinical areas increases, it is important that education programs to support their safe and 
470 Fredericus HJ van Loon et al Establishing the required components for training in US-guided peripheral intravenous cannulation

appropriate use are developed [36]. First attempt success logically increases after time and practice [17,27].

\section{Discussions}

In this systematic review, we focused on different training modules and components in use for ultrasoundguided peripheral intravenous cannulation in hospitalized adults, and determined that an optimal training session includes a didactic training session, followed by a handson session in a simulated environment, and is completed after a life-case training. The didactic session should cover topics as basic ultrasound physics, ultrasound-guided techniques of cannulation, and the upper extremity anatomy. The following 1:1 hands-on training session should focus on familiarization with ultrasound equipment and techniques using a nonhuman tissue model or on a life model without cannulating. A final life-case training gives participants the opportunity to gain experience and routine in cannulating veins on the upper extremity with an ultrasound-guided technique, under direct supervision, in live patients. Competency in ultrasound-guided peripheral intravenous cannulation can be achieved after following a dedicated training and closed supervision in a fixed curriculum, in which different steps regarding the procedure should consecutively be addressed, which could be a guidebook for the training of this procedure of ultrasound-guided peripheral intravenous cannulation.

According to the practice guideline for the use of ultrasound to guide vascular access procedures of the American Institute of Ultrasound in Medicine, training should include principles and practice of ultrasound, instruction in the techniques of ultrasound guidance for vascular access, and assessment of competency in a simulated or actual patient care setting $[8,37]$. In agreement with Ault et al competency can be defined as: being able to select the correct ultrasound probe; to set the correct depth, gain and target vein; properly positioning the target vein in the center of the ultrasound screen; correctly aligning the catheter tip in the middle of the probe over the target vein; tracking the catheter tip through the skin and tissue to the vein wall, including indenting the vein under ultrasound guidance before cannulating it; and cannulating the vein successful and achieving a bulls-eye image [16].

A combination of approaches of education to assist practitioners involved in the training for ultrasoundguided intravenous cannulation, increases the likelihood of transition of the procedure into practice [38]. Trainees became more competent when face-to-face and online learning were combined, which created the challenge of finding innovative ways to teach clinical skills [39]. Simulation-based training is one of the strategies used to improve quality in healthcare. The effect of simulationbased training for nurses seems to be positive in improving nurses' skills, whereas computer-based simulation is stated to be the most effective strategy on nurses' knowledge. Virtual reality training environments are used when training in reality is challenging because of the high costs, danger, time or effort involved, or when practicing on life cases is not possible due to ethical reasons [40]. Screenbased virtual reality simulators or virtual worlds are the most frequently used systems, and seem to be usable by a variety of end users, regardless of computer or gaming experience $[41,42]$. In the end, preparing trainees to bring their expertise to improve new processes requires learner activation [43].

Being a relatively new procedure, there is a limited amount of evidence to guide best training practices when teaching ultrasound-guided peripheral intravenous catheter insertion to novices. The practitioner responsible for ultrasound-guided intravenous access may vary based on the institution and the vein to be accessed, but may include technicians, nurses, advanced practice clinicians, and physicians [ 44]. Ultrasound-guided intravenous cannulation is an assessable procedure, because it is relatively easy to learn and could be provided at bed side [7,45-48]. Aside from the supplies necessary for vascular access, an ultrasound machine with a high-frequency capable probe is all that is necessary [44]. First attempt peripheral intravenous cannulation success would, however, be improved if practitioners with greater procedural experience and an increased perception of the likelihood of success performes the cannulation. [49]. The use of ultrasound should be considered early if peripheral venous cannulation proves to be difficult [ 50]. Nonetheless, patient-related factors, ultrasound physics and transducer properties should be part of the training for understanding of ultrasound related artefacts and pitfalls [2,5,51].

Selecting an appropriate vein to cannulate by scanning the upper extremity with the ultrasound probe after applying vasodilation is an important part of the procedure of ultrasound-guided peripheral intravenous cannulation [52]. Therefore, practitioners should have knowledge of upper extremity vascular anatomy before cannulating a vein and to distinguish veins from other structures $[10,14,15,19,21,22,27,52]$. Setting the correct gain and depth assists in creating an appropriate presentation of the target vein on the screen: knowledge of knobology of the ultrasound machine is required [22,52]. Knobology can be translated as the functionality of controls on an instrument as relevant to their application [53]. Becoming familiar with the machine and the controls used for image generation optimizes the scans being performed and enhances the use of ultrasound in patient 
care, although new-developed ultrasound machines automatically optimizes screen settings [53]. In addition, training should focus on hand-eye coordination, which is denoted as being the most difficult aspect of utilizing ultrasound [54]. Hand-eye coordination is necessary to hold the probe in one hand and cannulate the vessel with the other, but a lack in this practice can nullify any time saved during the procedure [55].

\section{Limitations}

A systematic review provides an objective overview of available evidence. Despite this, a number of challenges has to be taken into account when including qualitative studies. Data in qualitative research often comes from different sources, resulting in large quantities of raw material to be analyzed that must be systematically dissected, rearranged, organized and interpreted in order to answer the research questions [56]. Due to the inclusion of qualitative data sources, a quantitative data analysis with pooling of results was not possible [56,57]. Except for a lack of quantitative analyses, there were differences between the included studies themselves. Studies were carried out in different healthcare settings, including the emergency department, skills laboratory, postsurgical and medical units, and the surgical theatre complex. Moreover, various types of practitioners participated in the included studies, including emergency department nurses, technicians and physicians, registered nurses, nurse anesthetists, and medical students, with a different level of experience and knowledge. As with most invasive procedures, there is operator variability in skills and results, which possibly caused heterogeneity among the studies $[52,57]$. The effect of heterogeneity could be enhanced by the different approaches of ultrasound guidance that were applied throughout the studies, including the short- and long-axis approach, as each approach has its advantage, but also its limitations. In addition to the discussion on the most optimal approach, differences in training settings could have resulted in different outcomes. Neither identification of patients at high risk nor the definition of a difficult intravenous access was equal between the studies included. Finally, there may be publication bias in this systematic literature review, although the likelihood of publication bias was minimized by performing an extensive literature search for published and unpublished articles [57,58].

\section{Further research}

Although ultrasound machine size and equipment have evolved, the basic principles and fundamental functions have remained essentially the same. There is currently no consensus on the number of attempts that is required to determine competency, ranging from 5 to 25 attempts throughout the included studies [16,24,27,34,35,52,59].
Neither was there consensus on the definition of competency, resulting in the need for more data to determine the number of ultrasound-guided cannulations to become competent in this modality [52]. Larger studies, with an increased level of evidence, should decide which training curriculum results in highly skilled practitioners in ultrasound-guided peripheral intravenous cannulation. To add to this, differences between healthcare providers, as well as their different levels of experience and knowledge, should be part of this studies. While various training courses exist, most are focused on how to improve the visibility of peripheral veins on phantoms or in healthy volunteers. These enhanced simulation training should focus on pitfalls related to visualization by ultrasound, avoidance of applying excessive pressure on the tissue, and appropriate techniques to improve venous conditions and making them suitable for cannulation. Improved simulation training with a focus on needle manipulation and cannulation, that is adequately supported by evidence, could enhance the safety and efficacy of ultrasound-guided peripheral intravenous cannulation in hospitalized patients.

\section{Conclusions}

Training in and knowledge of ultrasound physics are important issues before applying ultrasound-guided procedures. Ultrasound physics and transducer properties introduce limitations with respect to beam width and elevation plane, that are especially important in case of small targets, such as peripheral vessels. Competency on ultrasound-guided peripheral intravenous cannulation can be achieved after following a brief training in a fixed curriculum. This study suggests evidence-based recommendations that provide directions for establishing consistency in the development of training programs and measuring competency through completion of a didactic training session, followed by a hands-on session in a simulated environment, and is completed after a supervised life-case training. More research is necessary to establish stronger recommendations and clearer directives.

\section{Conflict of interest: none}

Acknowledgements: The authors thank the librarians of the Catharina Hospital for their support during the databases' search.

\section{References}

1. Cooke M, Ullman AJ, Ray-Barruel G, Wallis M, Corley A, Rickard CM. Not "just” an intravenous line: Consumer perspectives on peripheral intravenous cannulation (PIVC). An 
international cross-sectional survey of 25 countries. PLoS One 2018; 13:e0193436.

2. Zingg W, Pittet D. Peripheral venous catheters: an underevaluated problem. Int J Antimicrob Agents 2009;34 Suppl 4:S38-S42.

3. Yalcinli S, Akarca FK, Can O, Sener A, Akbinar C. Factors affecting the first-attempt success rate of intravenous cannulation in older people. J Clin Nurs 2019;28:2206-2213.

4. Ozkula U, Özhasenekler A, Kurtoğlu Çelik G, et al. Tissue adhesives to secure peripheral intravenous catheters: A randomized controlled trial in patients over 65 years. Turkish J Emerg Med 2019;19:12-15.

5. van Loon FHJ, van Hooff LWE, de Boer HD, et al. The Modified A-DIVA Scale as a Predictive Tool for Prospective Identification of Adult Patients at Risk of a Difficult Intravenous Access: A Multicenter Validation Study. J Clin Med 2019;8:144.

6. van Loon FHJ, Buise MP, Claassen JJF, Dierick-van Daele AT, Bouwman ARA. Comparison of ultrasound guidance with palpation and direct visualisation for peripheral vein cannulation in adult patients: a systematic review and metaanalysis. Br J Anaesth 2018;121:358-366.

7. Gianstefani S, Catibog N, Whittaker AR, et al. Pocket-size imaging device: effectiveness for ward-based transthoracic studies. Eur Heart J Cardiovasc Imaging 2013;14:11321139.

8. Moore CL. Ultrasound first, second, and last for vascular access. J Ultrasound Med 2014;33:1135-1142.

9. van Loon FHJ, Scholten HJ, Korsten HHM, Bouwman ARA. Letter to the Editor on the original article "Ultrasound-guided peripheral intravenous access placement for children in the emergency department" by "Takehito Otani". Eur J Pediatr 2019;178:431-432.

10. Partovi-Deilami K, Nielsen JK, Moller AM, Nesheim SS, Jorgensen VL. Effect of Ultrasound-Guided Placement of Difficult-to-Place Peripheral Venous Catheters: A Prospective Study of a Training Program for Nurse Anesthetists. AANA J 2016;84:86-92.

11. Moureau N, Lamperti M, Kelly LJ, et al. Evidence-based consensus on the insertion of central venous access devices: definition of minimal requirements for training. Br J Anaesth 2013;110:347-356.

12. Lamperti M, Bodenham AR, Pittiruti M, et al. International evidence-based recommendations on ultrasound-guided vascular access. Intensive Care Med 2012;38:1105-1117.

13. Shamseer L, Moher D, Clarke M, et al. Preferred reporting items for systematic review and meta-analysis protocols (PRISMA-P) 2015: elaboration and explanation. BMJ 2015;350:g7647.

14. Singh J. Mendeley: A free research management tool for desktop and web. J Pharmacol Pharmacother 2010;1:62-63.

15. Edwards C, Jones J. Development and Implementation of an Ultrasound-Guided Peripheral Intravenous Catheter Program for Emergency Nurses. J Emerg Nurs 2018;44:33-36.

16. Ault MJ, Tanabe R, Rosen B. Peripheral Intravenous Access Using Ultrasound Guidance: Defining the Learning Curve. J Assoc Vasc Access 2015;20(1):32-36.
17. Duran-Gehring P, Bryant L, Reynolds JA, Aldridge P, Kalynych CJ, Guirgis FW. Ultrasound-guided peripheral intravenous catheter training results in physician-level success for emergency department technicians. J Ultrasound Med 2016;35:2343-2352.

18. Feinsmith S, Huebinger R, Pitts M, Baran E, Haas S. Outcomes of a Simplified Ultrasound-Guided Intravenous Training Course for Emergency Nurses. J Emerg Nurs 2018;44:169-175.e2.

19. Oliveira L, Lawrence M. Ultrasound-Guided Peripheral Intravenous Access Program for Emergency Physicians, Nurses, and Corpsmen (Technicians) at a Military Hospital. Mil Med 2016;181:272-276.

20. Owens TS, Nomura JT, Papas MA, Poole C, Marco D. Alteration of timing of nursing continuing education units for ultrasound-guided intravenous education does not increase utilization. Am J Emerg Med 2016;34:2248-2249.

21. Schoenfeld E, Boniface K, Shokoohi H. ED technicians can successfully place ultrasound-guided intravenous catheters in patients with poor vascular access. Am J Emerg Med 2011;29:496-501.

22. Shokoohi H, Boniface K, McCarthy M, et al. Ultrasoundguided peripheral intravenous access program is associated with a marked reduction in central venous catheter use in noncritically ill emergency department patients. Ann Emerg Med 2013;61:198-203.

23. Stolz LA, Cappa AR, Minckler MR, et al. Prospective evaluation of the learning curve for ultrasound-guided peripheral intravenous catheter placement. J Vasc Access 2016;17:366-370.

24. Weiner SG, Sarff AR, Esener DE, et al. Single-operator ultrasound-guided intravenous line placement by emergency nurses reduces the need for physician intervention in patients with difficult-to-establish intravenous access. J Emerg Med 2013;44:653-660.

25. White A, Lopez F, Stone P. Developing and sustaining an ultrasound-guided peripheral intravenous access program for emergency nurses. Adv Emerg Nurs J 2010;32:173188.

26. Dargin JM, Rebholz CM, Lowenstein RA, Mitchell PM, Feldman JA. Ultrasonography-guided peripheral intravenous catheter survival in ED patients with difficult access. Am J Emerg Med 2010;28:1-7.

27. Vitto MJ, Myers M, Vitto CM, Evans DP. Perceived Difficulty and Success Rate of Standard Versus UltrasoundGuided Peripheral Intravenous Cannulation in a Novice Study Group: A Randomized Crossover Trial. J Ultrasound Med 2016;35:895-898.

28. Miles G, Salcedo A, Spear D. Implementation of a successful registered nurse peripheral ultrasound-guided intravenous catheter program in an emergency department. J Emerg Nurs 2012;38:353-356.

29. Keleekai NL, Schuster CA, Murray CL, et al. Improving Nurses' Peripheral Intravenous Catheter Insertion Knowledge, Confidence, and Skills Using a Simulation-Based Blended Learning Program: A Randomized Trial. Simul Healthc 2016;11:376-384. 
30. Bahl A, Pandurangadu A, Tucker J, Bagan M. A randomized controlled trial assessing the use of ultrasound for nurse-performed IV placement in difficult access ED patients. Am J Emerg Med 2016;34:1950-1954.

31. Ortega R, Sekhar P, Song M, Hansen CJ, Peterson L. Videos in clinical medicine. Peripheral intravenous cannulation. N Engl J Med 2008;359:e26.

32. Bauman M, Braude D, Crandall C. Ultrasound-guidance vs. standard technique in difficult vascular access patients by ED technicians. Am J Emerg Med 2009;27:135-140.

33. Carter T, Conrad C, Wilson JL, Dogbey G. Ultrasound Guided Intravenous Access by Nursing versus Resident Staff in a Community Based Teaching Hospital: A "Noninferiority" Trial. Emerg Med Int 2015;2015:563139.

34. Adhikari S, Schmier C, Marx J. Focused simulation training: emergency department nurses' confidence and comfort level in performing ultrasound-guided vascular access. J Vasc Access 2015;16:515-520.

35. Moore C. An Emergency Department Nurse-Driven Ultrasound-Guided Peripheral Intravenous Line Program. J Assoc Vasc Access 2013;18:45-51.

36. Davis J, Faust T, Tajani A, et al. A randomized study of training with large versus small vessel size on successful ultrasound-guided peripheral venous access. J Vasc Access 2017;18:163-166.

37. Lian A, Rippey JCR, Carr PJ. Teaching medical students ultrasound-guided vascular access - which learning method is best? J Vasc Access 2017;18:255-258.

38. AIUM Practice Parameter for the Use of Ultrasound to Guide Vascular Access Procedures. J Ultrasound Med 2019;38:E4-E18.

39. Hayes C. Approaches to continuing professional development: putting theory into practice. Br J Nurs 2016;25:860864.

40. Hegland P, Aarlie H, Strømme H, Jamtvedt G. Simulationbased training for nurses: Systematic review and meta-analysis. Nurse Educ Today 2017;54:6-20.

41. Moskaliuk J, Bertram J, Cress U. Training in virtual environments: putting theory into practice. Ergonomics 2013;56:195-204.

42. Bracq MS, Michinov E, Jannin P. Virtual Reality Simulation in Nontechnical Skills Training for Healthcare Professionals: A Systematic Review. Simul Healthc 2019;14:188-194.

43. Polivka BJ, Anderson S, Lavender SA, et al. Efficacy and Usability of a Virtual Simulation Training System for Health and Safety Hazards Encountered by Healthcare Workers. Games Health J 2019;8:121-128.

44. Jones-Schenk J. Designing Education for Learning Activation. J Contin Educ Nurs 2017;48:539-540.

45. Currie M, Huecker M. Ultrasound Intravascular Access. In: Treasure Island (FL); 2019.
46. Panebianco NL, Fredette JM, Szyld D, Sagalyn EB, Pines JM, Dean AJ. What you see (sonographically) is what you get: vein and patient characteristics associated with successful ultrasound-guided peripheral intravenous placement in patients with difficult access. Acad Emerg Med 2009;16:1298-1303.

47. Tuffaha HW, Rickard CM, Webster J, et al. Cost-effectiveness analysis of clinically indicated versus routine replacement of peripheral intravenous catheters. Appl Health Econ Health Policy 2014;12:51-58.

48. McCarthy ML, Shokoohi H, Boniface KS, et al. Ultrasonography Versus Landmark for Peripheral Intravenous Cannulation: A Randomized Controlled Trial. Ann Emerg Med 2016;68:10-18.

49. Morata L, Ogilvie C, Yon J, Johnson A. Decreasing Peripherally Inserted Central Catheter Use With UltrasoundGuided Peripheral Intravenous Lines: A Quality Improvement Project in the Acute Care Setting. J Nurs Adm 2017;47:338-344.

50. Carr PJ, Rippey JC, Budgeon CA, Cooke ML, Higgins N, Rickard CM. Insertion of peripheral intravenous cannulae in the Emergency Department: factors associated with first-time insertion success. J Vasc Access 2016;17:182190.

51. Bodenham Chair A, Babu S, Bennett J, et al. Association of Anaesthetists of Great Britain and Ireland: Safe vascular access 2016. Anaesthesia 2016;71:573-585.

52. Rippey JC, Carr PJ, Cooke M, Higgins N, Rickard CM. Predicting and preventing peripheral intravenous cannula insertion failure in the emergency department: Clinician "gestalt" wins again. Emerg Med Australas 2016;28:658665.

53. Parker SI, Benzies KM, Hayden KA, Lang ES. Effectiveness of interventions for adult peripheral intravenous catheterization: A systematic review and meta-analysis of randomized controlled trials. Int Emerg Nurs 2017;31:15-21.

54. Enriquez JL, Wu TS. An introduction to ultrasound equipment and knobology. Crit Care Clin 2014;30:25-45.

55. Blaivas M, Lyon M. The effect of ultrasound guidance on the perceived difficulty of emergency nurse-obtained peripheral IV access. J Emerg Med 2006;31:407-410.

56. van Staa A, Evers J. "Thick analysis": strategie om de kwaliteit van kwalitatieve data-analyse te verhogen. KWALON 2014;43:43.

57. DerSimonian R, Laird N. Meta-analysis in clinical trials. Control Clin Trials. 1986;7:177-188.

58. Da Costa BR, Jüni P. Systematic reviews and meta-analyses of randomized trials: Principles and pitfalls. Eur Heart J 2014;35:3336-3345.

59. Dettori J. The random allocation process: two things you need to know. Evid Based Spine Care J 2010;1:7-9. 